\title{
P04-5-22 Poster session
}

\section{Transforming Growth Factor Induces Collagen Expression in Human Nasal Mucosa-derived Fibroblasts: Role of Connective Tissue Growth Factor Induction}

\author{
Wen-Bin Wu', Jiunn-Min Shieh ${ }^{2,3}$, Yih-Jeng Tsai ${ }^{1,4}$ \\ ${ }^{I}$ School of Medicine, Fu Jen Catholic University, Taiwan, ${ }^{2}$ Department of Internal Medicine, Chi-Mei Medical Center, \\ Tainan, Taiwan, ${ }^{3}$ Department of Recreation and Healthcare Management, Chia Nan University of Pharmacy and \\ Science, Tainan, Taiwan, ${ }^{4}$ Department of Otolaryngology Head and Neck Surgery, Shin Kong Wu Ho-Su Memorial \\ Hospital, Taipei, Taiwan
}

Chronic rhinosinusitis (CRS) is a common chronic disease involving the mucosa of the nasal cavity and sinuses. The disease can be classified into CRS with nasal polyps (CRSwNP) and without nasal polyps (CRSsNP). CRSsNP accounts for the majority of CRS cases $(\sim 60 \%)$ and is characterized by nasal fibrosis and basement membrane thickening. Recent evidence suggests that transforming growth factor (TGF) is involved in both the inflammatory and the remodeling component of respiratory airway disease, but how TGF exerts its effects on remodeling during CRSsNP has not yet been fully understood. In this study, the immunohistochemistry of CRSsNP and control normal mucosa was first examined, which revealed that TGF, collagen, and connective tissue growth factor (CTGF) were highly expressed in the CRSsNP mucosa than in the control. Therefore, the effects of TGF was further investigated on human CRSsNP mucosa-derived fibroblasts (NMDFs). Treatment of the NMDFs with TGF caused collagen expression, as determined by Western blotting, Sircol assay, and flowcytometric analysis. TGF induced CTGF mRNA and protein expression, subsequently causing CTGF secretion into the NMDF cultured medium. Meanwhile, addition of CTGF could lead to collagen expression in the NMDFs, suggesting that TGF induces collagen expression through CTGF induction. TGF-induced collagen and CTGF expression could be blocked by the DNA transcription and smad2/3 signaling inhibitors. In line with these observations, TGF enhanced smad2/3 phosphorylation and induced them translocation from cytosol into nucleus. The siRNA knockdown analysis confirmed that CTGF induction was necessarily required for TGF-induced collagen expression in the NMDFs. Collectively, our study provides the first evidence showing TGF, CTGF, and collagen expression in nasal mucosa from human CRSsNP patients. We also elucidate the signaling pathway required for TGFinduced collagen expression in human nasal mucosa-derived fibroblasts. Our study, at least, partly explains that TGF plays a certain role in tissue remodeling in/during CRSsNP development and progression. 